\title{
Normal Reference Range of Lateral Ventricle Parameters in Preterm Neonates by Ultrasonography
}

\author{
Sina Karamimagham, ${ }^{1}$ Maryam Poursadeghfard, ${ }^{2}$ and Fariba Hemmati ${ }^{3,}{ }^{*}$ \\ ${ }^{1}$ Fellowship of Neonatology, Department of Pediatrics, Medical School, Shiraz University of Medical Sciences, Shiraz, IR Iran \\ ${ }^{2}$ Assistant Professor of Neurology, Clinical Neurology Research Center, Department of Neurology, Medical School, Shiraz University of Medical Sciences, Shiraz, IR Iran \\ ${ }^{3}$ Associate Professor of Neonatology, Neonatal Research center, Department of Pediatrics, Shiraz University of Medical Sciences, Shiraz, IR Iran \\ "Corresponding author: Fariba Hemmati, MD, Department of Pediatrics, Neonatal Research Center, Shiraz University of medical Science, Shiraz, IR Iran. Tel/Fax: +98-7136122218; \\ +98-9171110281, E-mail: hemmatif@sums.ac.ir
}

Received 2016 November 18; Revised 2017 April 05; Accepted 2017 May 31.

\begin{abstract}
Background: Cranial ultrasound is considered as the method of choice for early evaluation of neonatal ventricular system. Since premature neonates are susceptible to intra-ventricular hemorrhage and hydrocephaly, it is essential to have normal values of parameters as reference ranges.

Objectives: To provide native information of ventricular sizes in normal premature infants in our area for screening policy. Methods: This Multicenter prospective study was conducted on normal premature babies with a gestational age of 26 to 35 weeks, from February to September 2014, in the South of Iran. Patients were divided into 10 groups according to their weeks of gestational age and each group was separately studied during the first 3 days of life. Statistical package for the Social Sciences version 16.0 software package [SPSS Inc, Chicago, Illinois] was used for statistical analysis. Continuous variables were described by mean with standard deviations (SD) and categorical variables by numbers and percentage appropriately. A 95\% confidence interval was supposed for all ventricular parameters (5,50 and 95 percentile). Each parameter that was above the 95 percentile for GA or birth weight was considered abnormal. Comparison between groups was done by Pearson correlation and Mann-Whitney and Wilcoxon Signed Ranks tests as appropriate. P value $\leq 0.05$ was regarded as statistically significant.

Results: 177 premature neonates were evaluated. The mean of ventricular index was $11.7 \mathrm{~mm} \pm 1.5 \mathrm{SD}$. The mean of anterior horn width and thalamo-occipital distance were $1.8 \mathrm{~mm} \pm 0.7 \mathrm{SD}$ and $15 \mathrm{~mm} \pm 2.7 \mathrm{SD}$, respectively. Ventricular index was gradually increased as the gestational age and birth weight increased, however, there was no obvious relationship between these and 2 other dimensions. There was no significant difference in dimensions between boys and girls, and also between normal vaginal delivery and cesarean section.

Conclusions: This study provides native normal reference values for lateral ventricular size in preterm infants in Iran. It helps physicians better understand the normal and abnormal size of the lateral ventricle in preterm neonates and improves both early diagnostic and therapeutic approaches.
\end{abstract}

Keywords: Cranial Ultrasound, Ventricular Index, Anterior Horn Width, Thalamo-Occipital Distance, Premature Neonate

\section{Background}

Delivery of live preterm neonates increased in recent decades. These neonates are susceptible to many complications. Intraventricular hemorrhage (IVH) is one of the major complications of prematurity (1). The majority of the patients with IVH are asymptomatic, however, among the survivors of severe IVH, long term consequences including post hemorrhagic hydrocephalus, seizures, paralysis, cerebral palsy, and other neurological deficits are seen $(1,2)$ About one third of neonates with IVH develop progressive ventricular dilation and hydrocephaly and $15 \%$ of them need ventriculoperitoneal shunt) VPS (to control raised intracranial pressure (3). Early diagnosis and management of IVH, due to significant side effects of VPS (infection, malfunction), is important. It is possible based on bedside screening Cranial Ultra Sonography) CUS (1). CUS, in the first week of life, is the best diagnostic tool as a screening test, when most hemorrhagic events occur. It is also a device of choice for post IVH follow-up (4). Accurate diagnosis of ventricular abnormality in preterm neonates is based on a good comprehension of normal and abnormal values, and it saves the time for rapid intervention. Available data on the reference curves are limited (5-11). Significant variation exists between the studies with respect to patient characteristics sample size, measured parameters, and timing of sonography. In most of the previous research, full term neonates are included $(7-9,11)$. A considerable number of studies were done more than a decade ago with less-sophisticated ultrasound equipment (5, 7-10). In Iran, there are no published studies regarding normal value of ventricular size in neonates. Variation in geno- 
type and phenotype among different populations may affect the size of lateral ventricle. We designed this study to define normal values of the size of lateral ventricles in preterm infants with a gestational age of 26 to 35 weeks in the Iranian population.

\section{Methods}

It was a multicenter prospective study in premature babies with GA of 26 to 35 weeks. All of these neonates were born or admitted in 3 teaching and public hospitals affiliated to the Shiraz University of Medical Science (Hafez, Hazrat Zeinaband and Nemazee hospitals), from February through September 2014, in Shiraz, the South of Iran. These 3 hospitals are the main academic and referral centers for high-risk pregnant mothers and have sub-special neonatal intensive care unit(NICU). Most of the patients admitted in these 3 NICUs are preterm neonates. NICU of Hafez, Hazrat Zeinab, and Nemazee Hospital has 10, 45, and 15 beds, respectively.

Neonates with unknown GA, anomaly within the central nervous system (brain and spinal cord), asphyxia, intrauterine infections, seizure, Intracranial, and intraventricular hemorrhage were excluded from the study.

CUS was done for all preterm infants with GA less than 35 weeks. Neonates were divided into 10 groups according to their weeks of GA and each group was separately studied.

All CUS was done by 1 expert neonatologist with a Mindray-M7 scanner and an ultrasonic transducer model P7-3s probe in parasagittal and coronal views through the anterior fontanel. For all newborns, gender, GA, birth weight, and route of delivery were recorded. Bilateral ventricular index (VI), anterior horn width (AHW), and thalamo-occipital distance (TOD) were measured.VI is the distance between the lateral wall of the anterior horn of the lateral ventricle and the falx cerebri in the coronal view at the level of the foramen of Monro, just anterior to the choroid plexus in the third ventricle. AHW is the oblique width of the anterior horn of the lateral ventricle at the widest point in the coronal plane. TOD is the distance between the external walls of the thalamus and lateral border of the occipital horn of the lateral ventricle in parasagittal plane (Figure 1). In order to increase the reliability and validity, $10 \%$ of measurements were done 2 times and under the supervision of a radiologist. The protocol of the study was approved by the local Ethics Committee of the university. Parents of all enrolled neonates were completely informed about the details of this study and informed consent was obtained verbally.
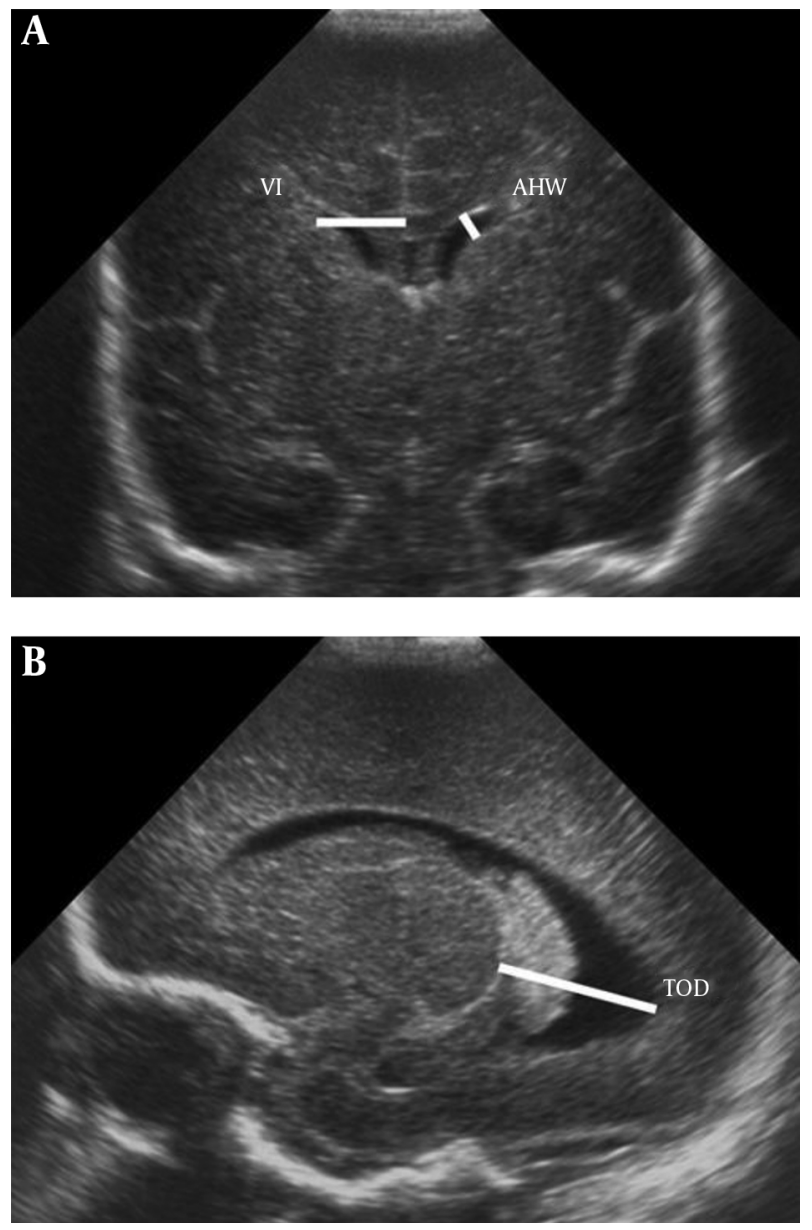

Figure 1. Measured Ventricular Parameters; A, Coronal View; B, Parasagittal View

\subsection{Statistical Analysis}

Statistical package for the social sciences version 16.0 software package [SPSS Inc, Chicago, Illinois] was used for statistical analysis. Continuous variables were described by mean with standard deviations (SD) and categorical variables by numbers and percentage appropriately. 95\% confidence interval was supposed for all ventricular parameters (5, 50 and 95 percentile). Each parameter passed the 95 percentile for GA or birth weight was considered abnormal. Comparison between groups was done by Pearson correlation and Mann-Whitney and Wilcoxon Signed Ranks tests as appropriate. P value $\leq 0.05$ was regarded as statistically significant.

\section{Results}

177 preterm neonates had the inclusion criteria. From these 177 neonates, 88 (49.7\%) were male and 89 (50.3\%) 
were female. 22 (12.4\%) and 155 (87.6\%) were born by normal vaginal delivery and cesarian section, respectively. There were 10 groups according to their GA. The most frequent group was neonates with GA 32 weeks (13.6\%) (Figure 2).

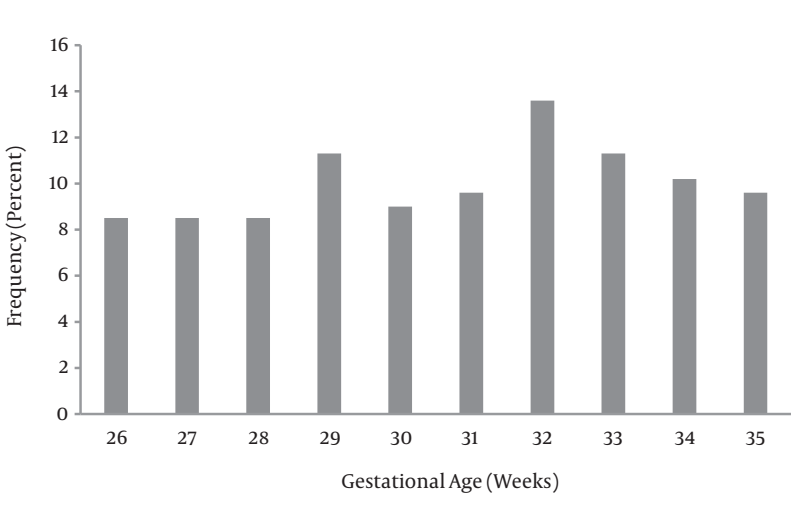

Figure 2. Frequency of 177 Premature Neonates According to Their Weeks of Gestational Age

Bilateral ventricular dimensions (VI, AHW, TOD) were measured for all cases and showed separately in each group to achieve a basic range for each GA. The maximum, minimum, and mean ranges of each dimension are shown in Tables 1 and 2.

Table 1. Intraventricular Dimensions of All Subjects

\begin{tabular}{lccc}
\hline & Minimum, mm & Mean \pm SD & Maximum, mm \\
\hline VI & 8 & $11.7 \pm 1.5$ & 17.9 \\
AHW & 0.6 & $1.8 \pm 0.7$ & 4.9 \\
TOD & 8.4 & $15 \pm 2.7$ & 22 \\
\hline
\end{tabular}

The nomogram with scatter plot for each of these items was drawn with $95 \%$ confidence interval. Each parameter that was above the 95th percentile for GA or birth weight was judged to have ventricular dilation.

VI showed a good linear correlation with GA, on the other hand, VI was increased as GA advanced ( $\mathrm{r}=0.45, \mathrm{PV}$ : $<0.001$ ) (Table 2, Figure 3).

However, AHW and TOD did not have any correlation with GA (r: -0.05 and -0.07, PV: 0.46 and 0.35 respectively) (Table 2, Figures 4 and 5 ).

Like GA, birth weight (BW) showed a good correlation with VI. When the BW increased, VI showed incremented pattern (r: 0.45, PV:< 0.001) (Table 2, Figure 6).

AHW and TOD did not have a constant relationship with weight (r: -0.06 and -0.07 , PV: 0.81 and 0.48 respectively) (Table 2, Figures 7 and 8)

The effect of gender and type of delivery on the ventricular parameters was evaluated. There was no significant

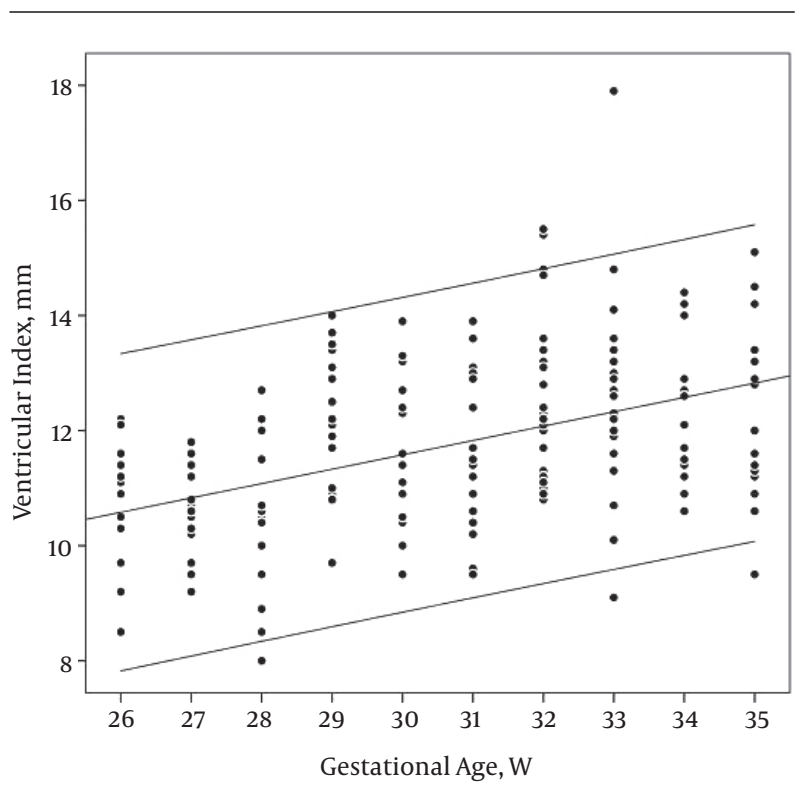

Figure 3. Correlation of Ventricular Index and Gestational Age Between 5 to 95 Percentile

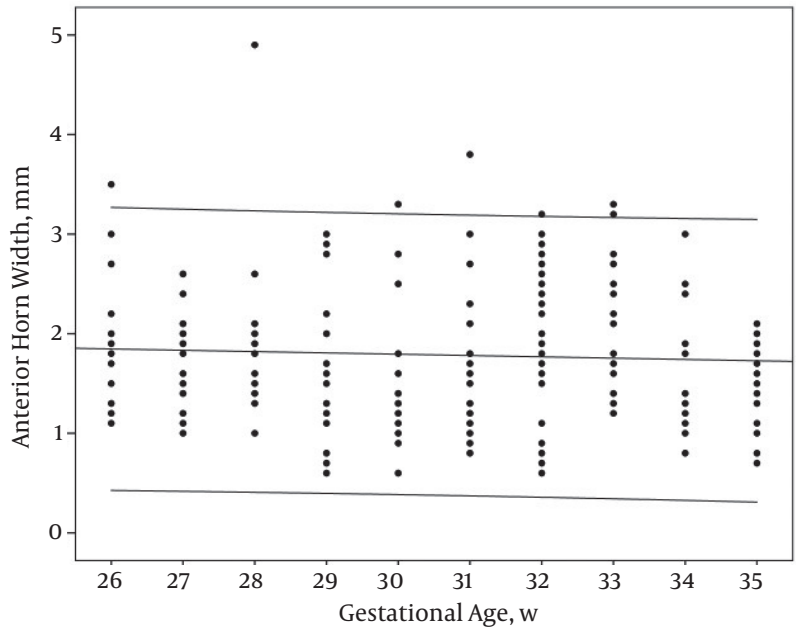

Figure 4. Correlation of the Anterior Horn Width and Gestational Age Between 5 to 95 Percentiles

difference in dimensions between boys and girls and also between normal vaginal delivery and cesarean section in all 10 gestational ages' groups $(\mathrm{P}>0.05)$.

Neonates with a GA of 33 weeks had a significant asymmetry in the VI of right $(11.6 \mathrm{~mm})$ and left $(13.2 \mathrm{~mm})$ side $(P=0.011)$. Indeed, in GA, 35 weeks AHW and TOD of the right and left side was significantly different $(\mathrm{P}=0.019$ and 0.026 , respectively). Among other GA, there was no notable asymmetry in size of both hemispheres (Table 3 ). 
Table 2. Intraventricular Dimensions of Each Gestational Age from 26 to 35 Weeks

\begin{tabular}{|c|c|c|c|c|c|c|c|c|c|}
\hline \multirow[t]{2}{*}{ GA, w } & \multicolumn{3}{|c|}{ VI } & \multicolumn{3}{|c|}{ AHW } & \multicolumn{3}{|c|}{ TOD } \\
\hline & $\operatorname{Min}$ & Mean & Max & Min & Mean & Max & Min & Mean & Max \\
\hline 26 & 8.5 & 10.4 & 12.2 & 1.1 & 1.9 & 2.3 & 10.1 & 15.9 & 20.9 \\
\hline 27 & 9.2 & 10.5 & 11.8 & 1.0 & 1.6 & 2.6 & 11.0 & 15.7 & 20.7 \\
\hline 28 & 8 & 10.3 & 12.7 & 1.0 & 1.9 & 4.9 & 10.3 & 15.3 & 19.4 \\
\hline 29 & 9.7 & 12.0 & 14.0 & 0.6 & 1.5 & 3 & 10.4 & 15.1 & 21.7 \\
\hline 30 & 9.5 & 11.6 & 13.9 & 0.6 & 1.6 & 3.3 & 8.4 & 14.6 & 19.7 \\
\hline 31 & 9.5 & 11.6 & 13.9 & 0.8 & 1.7 & 3.8 & 9.5 & 14.7 & 22 \\
\hline 32 & 10.8 & 12.5 & 15.5 & 0.6 & 1.9 & 3.2 & 11.2 & 15.5 & 20.1 \\
\hline 33 & 9.1 & 12.5 & 17.9 & 1.2 & 2.0 & 3.3 & 10.7 & 14.9 & 21.3 \\
\hline 34 & 10.6 & 12.3 & 14.4 & 0.8 & 1.6 & 3.0 & 11.9 & 14.2 & 20.0 \\
\hline 35 & 9.5 & 12.2 & 15.1 & 0.7 & 1.4 & 2.1 & 10.9 & 15.1 & 19.1 \\
\hline
\end{tabular}

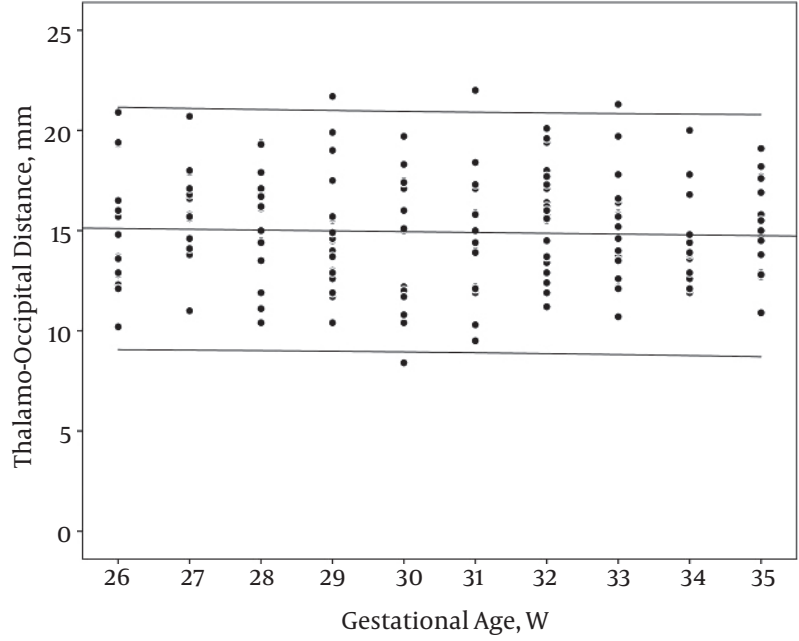

Figure 5. Correlation of Thalamo-Occipital Distance and Gestational Age Between 5 to 95 Percentiles

\section{Discussion}

CUS is the preferred method for evaluation of the neonatal ventricular system especially in premature neonates. Since premature neonates are very susceptible to IVH and post- IVH hydrocephaly, it helps to objectively assess the initial ventricular size and subsequent changes due to probable progressive ventricular enlargement in high risk infants. Therefore, CUS is used as a routine exam for preterm neonates with GA $<32$ weeks in most NICUs (12). To achieve an accurate assessment of ventricular size abnormality, it is essential to have normal values of parameters as reference ranges. Only a few millimeters of change in the ventricular size are enough to start closed observation and/or intervention.

This study provides new information regarding ventricular sizes in normal premature babies for screening

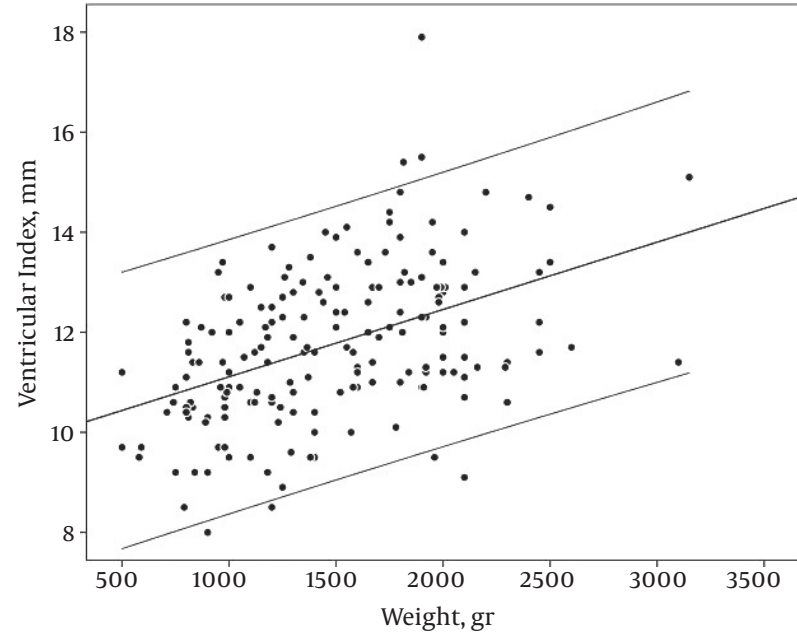

Figure 6. Correlation of Ventricular Index and Birth Weight Between 5 to 95 Percentiles

policy. In our study, we determined 3 normal sonographic parameters (VI, AHW, TOD) as reference values that could be measured easily in CUS and assessed with regarding the GA and birth weight. In spite of the most previous studies, we considered the birth weight as well as GA. The reference intervals for the VI presented in our study are slightly higher values than the curve published by Levene et al. (7) as well as Liao et al. (8). The lower transducer resolution 30 years ago may be responsible for the small difference between previous and this study. Our results showed that the VI considerably increased both with increase in GA and birth weight. Other studies also reported these results (7, $8,13,14)$. VI had a better relationship with BW rather than GA; it is shown that growth of brain tissue occurs parallel to weight gain rather than neonatal GA.

Mean of AHW (1.8 mm) is in line with the Liao et al. (8) study, however it is slightly higher than the Davies et al. (5) 


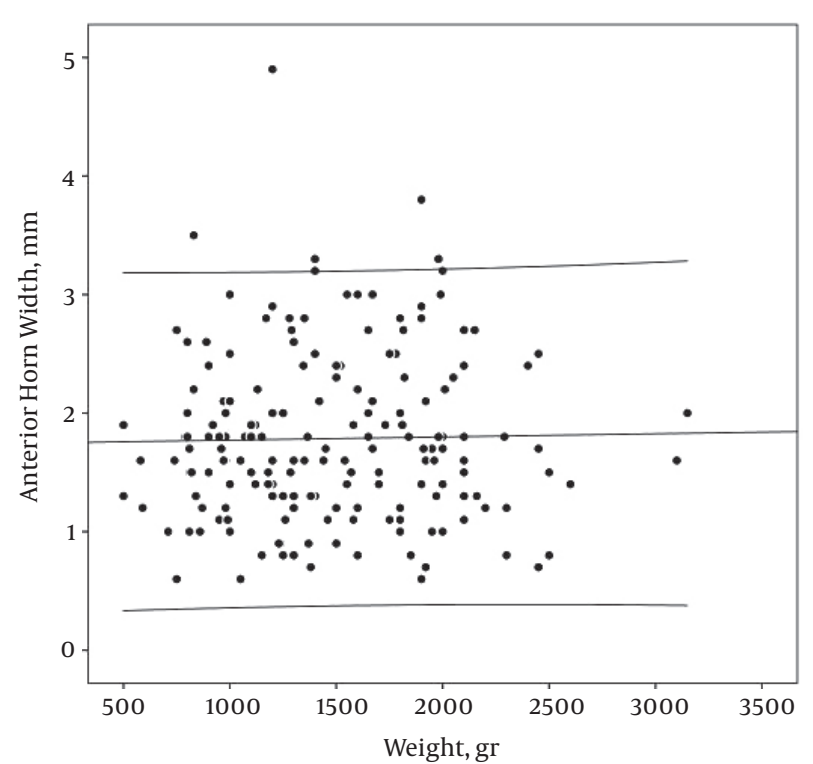

Figure 7. Correlation of the Anterior Horn Width and Birth Weight Between 5 to 95 Percentiles

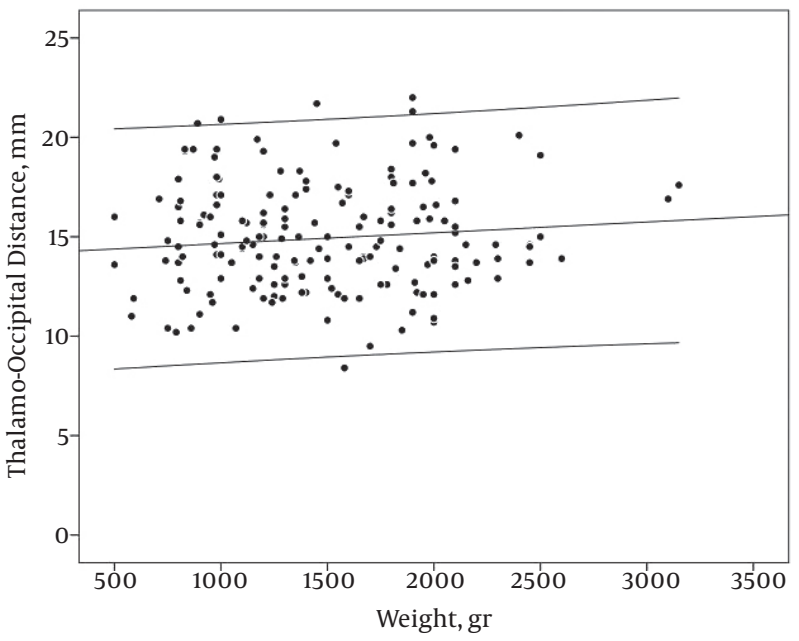

Figure 8. Correlation of the Thalamo-Ccipital Distance and Birth Weight Between 5 to 95 Percentiles

and Soudhi et al. (11) reports. Whether AHW correlated with GA is controversial, several reports $(5,8,13)$ similar to our results showed that AHW remained constant with increase in GA. Our study also showed just minimal change in AHW with birth weight.

In most of the reports $(5,8,11)$, the AHW is less than $3 \mathrm{~mm}$. It is in line with our results. Perry et al. (9) reported that AHW between 3 and $5 \mathrm{~mm}$ was not associated with neurodevelopmental impairment at a single follow
Table 3. Comparison of Dimensions in the Right and Left Hemispheres

\begin{tabular}{lccc}
\hline GA, w & Right and left VI & Right and left AHW & Right and left TOD \\
\hline $\mathbf{2 6}$ & 0.690 & 0.147 & 0.730 \\
$\mathbf{2 7}$ & 0.220 & 0.377 & 0.363 \\
$\mathbf{2 8}$ & 0.245 & 0.093 & 0.192 \\
\hline $\mathbf{2 9}$ & 0.064 & 0.183 & 0.737 \\
\hline $\mathbf{3 0}$ & 0.706 & 0.712 & 0.587 \\
\hline $\mathbf{3 1}$ & 0.406 & 0.171 & 0.246 \\
\hline $\mathbf{3 2}$ & 0.110 & 0.745 & 0.567 \\
\hline $\mathbf{3 3}$ & $0.011[$ Left $>$ Right] & 0.493 & 0.239 \\
\hline $\mathbf{3 4}$ & 0.123 & 0.553 & 0.663 \\
\hline $\mathbf{3 5}$ & 0.255 & $0.019[$ Left $>$ Right] & $0.026[$ Right $>$ Left] \\
\hline
\end{tabular}

up visit in 13 infants during the first year of life; however, Govaert et al. (15) reported that values exceeding $6 \mathrm{~mm}$, are associated with ventricular ballooning and suggest the need for treatment.

In our study, the mean of TOD was $15 \mathrm{~mm} \pm 2.7$ $\mathrm{mm}$. there was no obvious relationship between GA, birth weight, and TOD. There is controversy about the size of occipital horn and also whether normal values of occipital horn size depended on GA or not. Reeder et al. (10), who compared the size of occipital horn in normal premature infants and premature neonates with IVH or neural tube defect, concluded that an occipital horn size of more than $16 \mathrm{~mm}$ suggest intracranial pathology. Davies et al. (5) reported higher values, with an upper limit $24.7 \mathrm{~mm}$ but Sondhi et al. (11) showed a smaller occipital horn size. In our study, the maximum values were $22 \mathrm{~mm}$. The difference between reports may be due to difficulties in visualizing the occipital horn and obliquity of the transducer.

VI increased with gestational age and birth weight, however, AHW and TOD remained constant. It is interpreted that growth results from tissue substrate. On the other hand, with increasing the GA, the ratio of the brain parenchyma to the ventricular space is increased. Parameters like AHW, which are just inter-ventricular space, reflect only ventricular size without tissue component and do not show regular incremented pattern with increasing GA or BW. There are also similar reports regarding other inter-ventricular dimensions, such as third ventricle width, fourth ventricle width, and fourth ventricle length $(5,16)$. However, the Sondhi et al. study (11) demonstrated a marked increase in TOD and AHW with progression of GA.

There was no significant right and left ventricular asymmetry in our study except for VI (left > right; P value 
0.011) in GA 33 weeks and AHW (left > right; P value 0.019) and TOD (right $>$ left; Pvalue 0.026) dimensions in the 35th week of GA. Some studies showed minor ventricular asymmetry (17-19). Generally, the left side was reported a little larger than the right one and this asymmetry was more pronounce in the occipital horns. They assumed it could be associated with a larger choroid plexus in the left side (18, 19). We saw these differences in the ventricular size only in 2 groups ( 33 and 35 weeks). In 2 dimensions (VIs and AHWs), the left side was more dilated, however, in TOD the right side was larger. Nonetheless, we did not find a considerable asymmetry in all patients.

Ichihashi et al. evaluated 60 neonatal infants in the first and second weeks of birth. They found left ventricular prominence in most of their cases, but they concluded that it is the result of physiological individual differences. Their subjects had normal development in the 1 and 1/2year follow up (20). In a study done in 1998, Koeda and his colleague reported that brief asymmetry of the lateral ventricle in preterm neonates could be due to the position of the head during CUS. They supposed the soft brain and gravity might cause the shift of cerebrospinal fluid from the non-dependent to the dependent lateral ventricle and influence the results of CUS in a different position (21). In another study, Davies and co-workers could not establish this hypothesis and in their study, the head position had no effect on the measurement between non-dependent and dependent ventricle (5). We measured all sonographic dimensions in supine position and did not have a significant asymmetry in the right and left data like Davies results (5). There was no difference in size of lateral ventricle between both sexes in all gestational age and birth weight groups in this survey, however, there are some reports that demonstrated the large ventricular size after birth in the male sex, although it has been emphasized that its effect diminished in preterm infants (13).

In this study, the mode of delivery (normal vaginal delivery versus cesarean section) did not affect the ventricular parameters in all age and weight related groups. A survey on 59 full-term infants on the 3rd to 7th postpartum days showed that in addition, the vaginal or cesarean section delivery did not influence the ventricular size (22). However, other data gathered from the term Nigerian neonates showed the opposite result. In their study, vaginal delivery caused smaller lateral ventricular width at birth, which was not apparent at the 6th week of age. Therefore, they supposed it might be due to compression by the birth canal without pathological significance (23).

We repeated the measurement of ventricular dimension (VI, AHW, TOD) in 10\% of neonates. There was good intra and inter observer correlation. This study has some methodological limitations:
1) Short duration of study time.

2) Few patients with lower GA.

3) Use cross-sectional data, No follow up

\subsection{Conclusions}

CUS provides a convenient, safe, and rapid method for early measurement of ventricular system in premature neonates who are at risk for intra-and peri-ventricular hemorrhage. The results of this study provide a reference range of normal values in preterm infants from 26 to 35 weeks' GA. It was the first report in Iran. These values are as follow: minimum and maximum of VI: 8 and $17.9 \mathrm{~mm}$, minimum and maximum of AHW: 0.6 and $4.9 \mathrm{~mm}$, and minimum and maximum of TOD: 8.4 and $21.9 \mathrm{~mm}$. This native reference range helps physicians better understand the normal and abnormal size of the lateral ventricle in preterm neonates and improves both early diagnostic and therapeutic approaches.

Key massage: Variation in genotype and phenotype among different population may affect the size of lateral ventricle. We designed this study to define normal values of the size of lateral ventricles in preterm infants with the gestational age of 26 to 35 weeks in Iranian population.

\section{Acknowledgments}

The present article was extracted from the thesis written by Sina Karamimagham and was financially supported by the Shiraz University of Medical Sciences grant number 92-6658. The authors would like to thank the center for development of clinical research of Nemazee Hospital and Dr. Nasrin Shokrpour for editorial assistance as well as Dr Fariba Zarei, Assistant professor of Radiology of Shiraz University of Medical Sciences, for supervision of cranial ultrasonography.

\section{Footnote}

Conflicts of Interest: No conflicts of interest are declared by the authors.

\section{References}

1. Ballabh P. Intraventricular hemorrhage in premature in fants: mechanism of disease. Pediatr Res. 2010;67(1):1-8. doi 10.1203/PDR.ob013e3181c1b176. [PubMed: 19816235].

2. Kenet G, Kuperman AA, Strauss T, Brenner B. Neonatal IVHmechanisms and management. Thromb Res. 2011;127 Suppl 3:S120-2. doi: 10.1016/S0049-3848(11)70032-9. [PubMed: 21262430].

3. Murphy BP, Inder TE, Rooks V, Taylor GA, Anderson NJ, Mogridge N, et al. Posthaemorrhagic ventricular dilatation in the premature infant: natural history and predictors of outcome. Arch Dis Child Fetal Neonatal Ed. 2002;87(1):F37-41. [PubMed: 12091289]. 
4. Fallas SR. Brain ultrasound in intracranial hemorrhage and hypoxicischemic encephalopathy in high risk preterm patients. Ultrasound Med Biol. 2006;32(5):P43-4. doi:10.1016/j.ultrasmedbio.2006.02.148.

5. Davies MW, Swaminathan M, Chuang SL, Betheras FR. Reference ranges for the linear dimensions of the intracranial ventricles in preterm neonates. Arch Dis Child Fetal Neonatal Ed. 2000;82(3):F21823. [PubMed: 10794790].

6. Salomon LJ, Bernard JP, Ville Y. Reference ranges for fetal ventricular width: a non-normal approach. Ultrasound Obstet Gynecol. 2007;30(1):61-6. doi: 10.1002/uog.4026. [PubMed: 17506037].

7. Levene MI. Measurement of the growth of the lateral ventricles in preterm infants with real-time ultrasound. Arch Dis Child. 1981;56(12):900-4. [PubMed: 7332336].

8. Liao MF, Chaou WT, Tsao LY, Nishida H, Sakanoue M. Ultrasound measurement of the ventricular size in newborn infants. Brain Dev 1986;8(3):262-8. [PubMed: 3532852].

9. Perry RN, Bowman ED, Murton LJ, Roy RN, de Crespigny LC. Ventricular size in newborn infants. J Ultrasound Med. 1985;4(9):475-7. [PubMed: 3903200].

10. Reeder JD, Kaude JV, Setzer ES. The occipital horn of the lateral ventricles in premature infants. An ultrasonographic study. Eur J Radiol. 1983;3(2):148-50. [PubMed: 6873077].

11. Sondhi V, Gupta G, Gupta PK, Patnaik SK, Tshering K. Establishment of nomograms and reference ranges for intra-cranial ventricular dimensions and ventriculo-hemispheric ratio in newborns by ultrasonography. Acta Paediatr. 2008;97(6):738-44. doi: 10.1111/j.16512227.2008.00765.x. [PubMed: 18397357].

12. van Wezel-Meijler G, Steggerda SJ, Leijser LM. Cranial ultrasonography in neonates: role and limitations. Semin Perinatol. 2010;34(1):28-38. doi:10.1053/j.semperi.2009.10.002. [PubMed: 20109970].

13. Brouwer MJ, de Vries LS, Groenendaal F, Koopman C, Pistorius LR, Mulder EJ, et al. New reference values for the neonatal cerebral ventricles. Radiology. 2012;262(1):224-33. doi: 10.1148/radiol.11110334. [PubMed:
22084208].

14. Soni JP, Gupta BD, Soni M, Singh RN, Purohit NN, Gupta M, et al. Normal parameters of ventricular system in healthy infants. Indian Pediatr. 1995;32(5):549-55. [PubMed: 8613313].

15. Govaert P, De Vries LS. An Atlas of Neonatal Brain Sonography:(CDM 182-183). John Wiley \& Sons; 2010.

16. Saliba E, Bertrand P, Gold F, Vaillant MC, Laugier J. Area of lateral ventricles measured on cranial ultrasonography in preterm infants: reference range. Arch Dis Child. 1990;65(10 Spec No):1029-32. [PubMed: 2241221].

17. Horsch S, Bengtsson J, Nordell A, Lagercrantz H, Aden U, Blennow M. Lateral ventricular size in extremely premature infants: $3 \mathrm{D}$ MRI confirms 2D ultrasound measurements. Ultrasound Med Biol. 2009;35(3):360-6. doi: 10.1016/j.ultrasmedbio.2008.09.006. [PubMed: 19056162].

18. Batton DG, Swails TL. Isolated lateral ventricular asymmetry in very low-birth-weight infants: a left-sided lesion?. Am J Perinatol. 1998;15(3):183-6. doi: 10.1055/s-2007-993922. [PubMed: 9572374].

19. Enriquez G, Correa F, Lucaya J, Piqueras J, Aso C, Ortega A. Potential pitfalls in cranial sonography. Pediatr Radiol. 2003;33(2):110-7. doi: 10.1007/s00247-002-0836-y. [PubMed: 12557067].

20. Ichihashi K, Iino M, Eguchi Y, Uchida A, Honma Y, Momoi M. Difference between left and right lateral ventricular sizes in neonates. Early Hum Dev. 2002;68(1):55-64. [PubMed: 12191529].

21. Koeda T, Ando Y, Takashima S, Takeshita K, Maeda K. Changes in the lateral ventricle with the head position: ultrasonographic observation. Neuroradiology. 1988;30(4):315-8. [PubMed: 3050588].

22. Zhang WL. [Measurement of neonatal ventricular size]. Zhonghua Yi Xue Za Zhi. 1993;73(3):151-3. [PubMed: 8391911] 190-1.

23. Ebruke B, Tongo O, Sofoluwe A, Orimadegun B, Obajimi M, Akinyinka $O$. Intracranial ventricular sizes and correlates in term Nigerian infants at birth and six weeks. Internet J Pediatr Neonat. 2009;11(1). 